\title{
DIRECTION DE JULIA DE SYSTÈMES ET SOMME DE FONCTIONS NORMALES
}

\author{
NOBUSHIGE TODA
}

1. Introduction. Dans ce mémoire, on étend quelques résultats obtenus dans [10] au système et comme application, on considère sur la somme de fonctions normales.

Soient $D$ un domaine dans le plan $|z|<\infty, g_{0}(z), g_{1}(z), \cdots, g_{n}(z)$ $(n \geqq 1) n+1$ fonctions holomorphes dans $D$ n'ayant pas de zéros communs à toutes et $g=\left(g_{0}, \cdots, g_{n}\right)$ un système de ces $n+1$ fonctions dans $D$. On dit qu'une combinaison linéaire à coefficients constants

$$
a_{0} g_{0}(z)+a_{1} g_{1}(z)+\cdots+a_{n} g_{n}(z) \quad(\not \equiv 0)
$$

est

1) lacunaire dans $D$ si elle n'admet pas de zéro dans $D$;

2) exceptionnelle au sens de Picard dans $D$ si elle n'admet qu'un nombre fini de zéros dans $D$ au plus.

Soit $f=\left(f_{0}, \cdots, f_{n}\right)$ un système transcendant dans le plan $|z|<\infty$; c'est-à-dire, les fonctions $f_{0}, \cdots, f_{n}$ sont entières sans zéros communs à toutes et

$$
\lim _{r \rightarrow \infty} \frac{T(r, f)}{\log r}=\infty
$$

où $T(r, f)$ est la fonction caractéristique du système $f$ définie par Cartan [1].

DÉfinition 1 (Direction de Julia). On dit que $J: \arg z=\theta_{J}$ $\left(0 \leqq \theta_{J}<2 \pi\right)$ est une direction de Julia du système $f$ s'il n'y a qu'au plus $2 n$ combinaisons linéaires des fonctions $f_{0}, \cdots, f_{n}$, homogènes à coefficients constants, linéairement indépendantes $n+1$ à $n+1$ et exceptionnelles au sens de Picard dans $\Delta_{c}\left(\theta_{J}\right)=\left\{z ;\left|\arg z-\theta_{J}\right|<\varepsilon\right\}$, $\varepsilon$ étant positif quelconque.

Received December 21, 1970.

Revised December 15, 1971. 
Sur les fonctions exceptionnelles au sens de Julia que l'on utilise souvant, Ostrowski [7] et Lehto-Virtanen [5] étudient beaucoup de choses en détail.

On utilise les symboles usuels de la théorie de Nevanlinna-Selberg ([6], [8]) librement.

2. Systèmes à au moins une direction de Julia. Correspondant aux fonctions algébroïdes dans $|z|<\infty$ ([10]), on a le

THÉORÈME 1. Soit $f=\left(f_{0}, \cdots, f_{n}\right)$ un système transcendant dans le plan $|z|<\infty$. S'il y a au moins un rapport entre les fonctions $f_{0}, \cdots, f_{n}$ n'étant pas exceptionnel au sens de Julia, le système $f$ admet au moins une direction de Julia.

On peut démontrer facilement ce théorème en modifiant un peu la démonstration du théorème 1 dans [10].

LEMme. Soit $f=\left(f_{0}, \cdots, f_{n}\right)$ un système dans le plan $|z|<\infty$ où $f_{0}, \cdots, f_{n}$ sont entières. Alors, pour tout $i \neq j$, on $a$

$$
T\left(r, f_{i} / f_{j}\right)-O(1)<T(r, f)<\sum_{k \neq j} T\left(r, f_{k} / f_{j}\right)+O(1)
$$

où $f_{j} \not \equiv 0$ ([1], [9]).

Corollaire 1. Soit $f=\left(f_{0}, \cdots, f_{n}\right)$ un système transcendant dans le plan $|z|<\infty$ tel que

$$
\limsup _{r \rightarrow \infty} \frac{T(r, f)}{(\log r)^{2}}=\infty .
$$

Alors, le système $f$ admet au moins une direction de Julia.

En effet, d'après le lemme, il existe au moins un rapport $f_{i} / f_{j}$ tel que

$$
\limsup _{r \rightarrow \infty} \frac{T\left(r, f_{i} / f_{j}\right)}{(\log r)^{2}}=\infty
$$

Alors, grâce à un résultat dans [5], la fonction $f_{i} / f_{j}$ n'est pas exceptionnelle au sens de Julia. Donc, en vertu du théorème 1 , le système $f$ admet au moins une direction de Julia.

Corollaire 2. Soit $f=\left(1, f_{1}, \cdots, f_{n}\right)$ un système transcendant dans 
le plan $|z|<\infty$. Alors, le système $f$ admet au moins une direction de Julia.

En effet, d'après le lemme, il existe au moins une fonction $f_{i}$ $(1 \leqq i \leqq n)$ qui est transcendante. Par conséquent, la fonction $f_{i}=f_{i} / 1$ n'est pas exceptionnelle au sens de Julia [7]. Donc, grâce au théorème 1 , le système $f$ admet au moins une direction de Julia.

THÉORÈME 2. Soient $f=\left(f_{0}, \cdots, f_{n}\right)$ un système transcendant dans le plan $|z|<\infty$ à au moins une direction de Julia et $A$ une $(n+1, n+1)$ matrice régulière à éléments constants. Mettons

$$
\left(F_{0}, \cdots, F_{n}\right)^{t}=A\left(f_{0}, \cdots, f_{n}\right)^{t},
$$

alors, un nouveau système $F=\left(F_{0}, \cdots, F_{n}\right)$, qui est transcendant dans le plan $|z|<\infty$, admet au moins une direction de Julia.

En effet, soit $\arg z=\theta_{f}$ une direction de Julia de $f$. Alors, on peut prouver facilement qu'elle est aussi une direction de Julia de $F$.

N.B. 1. L'inverse du théorème 1 n'est pas valide en général. En effet, on peut donner facilement un exemple-contre en utilisant une fonction méromorphe dans $|z|<\infty$ qui est exceptionnelle au sens de Julia, mais admet au moins une direction de Julia, qui est donnée par Zinno [12].

3. Systèmes sans direction de Julia. Dans ce paragraphe, on recherche sur le système qui n'admet pas de direction de Julia.

THÉORÈme 3. Soient $f=\left(f_{0}, \cdots, f_{n}\right)$ un système transcendant dans le plan $|z|<\infty$ sans direction de Julia et

$$
F=\sum_{i=0}^{n} a_{i} f_{i} \quad(\not \equiv 0), \quad G=\sum_{i=0}^{n} b_{i} f_{i} \quad(\not \equiv 0)
$$

deux combinaisons linéaires à coefficients constants. Alors, le rapport $F / G$ est exceptionnel au sens de Julia.

Démonstration. Supposons que $F / G$ ne soit pas constante. Alors au moins une valeur des $a_{i} b_{j}-a_{j} b_{i}(i \neq j)$ n'est pas égale à zéro. On peut supposer que $a_{0} b_{1}-a_{1} b_{0} \neq 0$ et $f_{0} \neq \equiv, f_{1} \neq \equiv 0$. Soit 


$$
A=\left(\begin{array}{ccccc}
a_{0} & a_{1} & a_{2} & \cdots & a_{n} \\
b_{0} & b_{1} & b_{2} & \cdots & b_{n} \\
& & 1 & & 0 \\
& 0 & & \ddots & 1
\end{array}\right),
$$

alors $|A|=a_{0} b_{1}-a_{1} b_{0} \neq 0$ et

$$
\left(F, G, f_{2}, \cdots, f_{n}\right)^{t}=A\left(f_{0}, f_{1}, \cdots, f_{n}\right)^{t} .
$$

Par conséquent, d'après le théorème 2 le système $\left(F, G, f_{2}, \cdots, f_{n}\right)$ n'admet pas de direction de Julia. Cela veut dire que $F / G$ est exceptionnelle au sens de Julia en vertu du théorème 1.

CoRollaire 3. Soit $f$ un système comme dans le théorème 3. Alors, le système $f$ n'admet pas de combinaisons linéaires, homogènes à coefficients constants et exceptionnelles au sens de Picard, c'est-à-dire, chaque combinaison à coefficients constants

$$
F=\sum_{i=0}^{n} a_{i} f_{i} \quad(\not \equiv 0)
$$

admet le zéro une infinité dénombrable de fois.

Démonstration. Par l'hypothèse, tous les rapports entre les fonctions $f_{0}, \cdots, f_{n}$ sont exceptionnels au sens de Julia et d'après le lemme au moins un rapport est transcendant, de sorte que chaque fonction $f_{i}$ est transcendante ou égale à zéro identiquement. Il y a au moins deux fonctions transcendantes et elles admettent le zéro une infinité dénombrable de fois. Soit $F$ la combinaison donnée dans ce corollaire. Alors, d'après le théorème 3 , les fonctions

$$
F / f_{i} \quad\left(i ; a_{i} \neq 0, f_{i} \not \equiv 0\right)
$$

sont exceptionnelles au sens de Julia, de sorte que $F$ admet le zéro une infinité dénombrable de fois dans le plan $|z|<\infty$.

THÉORÈme 4. Soit $f=\left(f_{0}, \cdots, f_{n}\right)$ un système transcendant dans le plan $|z|<\infty$ sans direction de Julia tel qu'une des fonctions $f_{0}, \cdots, f_{n}$ (soit $f_{0}$ ) est un produit canonique. Alors, chaque combinaison ( $\left.\not \equiv 0\right)$ linéaire des fonctions $f_{0}, \cdots, f_{n}$, homogène à coefficients constants n'admet pas de valeurs asymptotiques finies en point à l'infini. 
Démonstration. D'après l'hypothèse, l'inégalité (voir [1])

$$
N\left(r, 0, f_{0}\right) \leqq T(r, f)+O(1)
$$

entraîne que l'ordre de $f_{0}$ est zéro. De plus, grâce au lemme, pour tout $i=1, \cdots, n$, on a

$$
T\left(r, f_{i}\right) \leqq T(r, f)+T\left(r, f_{0}\right)+O(1),
$$

de sorte que toutes les fonctions $f_{0}, \cdots, f_{n}$ sont d'ordre nul. Soit

$$
F=\sum_{i=0}^{n} a_{i} f_{i} \quad(\not \equiv 0)
$$

une combinaison linéaire à coefficients constants. Alors, $F$ est transcendante d'après le corollaire 3 . Si $F$ admet une valeur asymptotique finie, il faut que l'ordre de $F$ soit plus grand que $1 / 2$ d'après un resultat connu bien de Wiman. Mais, l'ordre de $F$ est nul parce que $f_{0}, \cdots, f_{n}$ sont d'ordre nul. Cela veut dire que $F$ n'admet pas de valeurs asymptotiques finies en point à l'infini.

DÉFINITION 2. Soient $f=\left(f_{0}, \cdots, f_{n}\right)$ un système transcendant dans le plan $|z|<\infty$ et

$$
F=a_{0} f_{0}+\cdots+a_{n} f_{n} \quad(\not \equiv 0)
$$

une combinaison linéaire à coefficients constants. On dit que $F$ est exceptionnelle au sens de Valiron si

$$
\Delta(F)=1-\liminf _{r \rightarrow \infty} \frac{N(r, 0, F)}{T(r, f)}>0 .
$$

THÉOṘ̀me 5. Soient $f=\left(f_{0}, \cdots, f_{n}\right)$ un système transcendant dans le plan $|z|<\infty$ sans direction de Julia et

$$
F=a_{0} f_{0}+\cdots+a_{n} f_{n} \quad(\not \equiv 0)
$$

une combinaison linéaire quelconque à coefficients constants. Alors, on a

$$
\Delta(F)=0 \text {. }
$$

Démonstration. On peut supposer que $a_{0} f_{0} \not \equiv 0$. D'après le théorème $3, F / f_{0}$ es exceptionnelle au sens de Julia et $F$ est transcendante. En appliquant un résultat d'Ostrowski [7] à $F / f_{0}$, il y a un nombre $K_{F}$ indépendant de $r$ tel que pour tout $r>0$ 


$$
\left|n(r, 0, F)-n\left(r, 0, f_{0}\right)\right|<K_{F},
$$

par conséquent, on a

$$
\left|N(r, 0, F)-N\left(r, 0, f_{0}\right)\right|<O(\log r),
$$

de sorte que $f$ étant transcendant

$$
\Delta(F)=\Delta\left(f_{0}\right) .
$$

Maintenant, considérons la fonction $w(z)$ définie par l'équation

$$
f_{0}(z) w^{n}+f_{1}(z) w^{n-1}+\cdots+f_{n}(z)=0 .
$$

Alors, d'après un résultat de Valiron [11], on a

$$
|T(r, w)-T(r, f) / n|<O(1)
$$

et

$$
N(r, w)=N\left(r, 0, f_{0}\right) / n
$$

Par conséquent, on a

$$
\liminf _{r \rightarrow \infty} \frac{N(r, w)}{T(r, w)}=\liminf _{r \rightarrow \infty} \frac{N\left(r, 0, f_{0}\right)}{T(r, f)}
$$

De plus, comme on a démontré dans [10], on peut donner dans notre cas

$$
\Delta(\infty, w)=0
$$

En conséquence, de (1) et (2), on a

$$
\Delta(F)=0 \text {. }
$$

Corollaire 4.

$$
\delta(F)=1-\limsup _{r \rightarrow \infty} \frac{N(r, 0, F)}{T(r, f)}=0 .
$$

N.B. 2. Ce théorème est une précision du corollaire 3 .

4. Somme de fonctions normales. Soient $h_{1}, h_{2}$ deux fonctions exceptionnelles au sens de Julia. On ne sait pas si la somme de deux fonctions

$$
h_{1}+h_{2}
$$


est exceptionnelle au sens de Julia en général. On donne ici une condition suffisante pour qu'une somme de fonctions exceptionnelle au sens de Julia le soit aussi.

Soient $g_{1}, \ldots, g_{n}(n \geqq 2) n$ fonctions transcendantes, exceptionnelles au sens de Julia et $g$ un produit canonique des pôles des fonctions $g_{1}$, $\cdots, g_{n}$. On met

$$
f_{0}=g, f_{1}=g g_{1}, \cdots, f_{n}=g g_{n} .
$$

Alors, les fonctions $f_{0}, \cdots, f_{n}$ sont entières sans zéros commnus à toutes. Dans ce cas, on a le

THÉORÈME 6. Si le système $f=\left(f_{0}, \cdots, f_{n}\right)$ n'admet pas de directions de Julia, alors

i) $\sum_{i=1}^{n} \alpha_{i} g_{i},\left(\alpha_{i}\right) \neq(0)$, est exceptionnelle au sens de Julia,

ii) pour tout $i \neq j, g_{i} / g_{j}$ est exceptionnelle au sens de Julia.

Démonstration. Considérons la combinaison

$$
F=f_{0}+\sum_{i=1}^{n} \alpha_{i} f_{i} .
$$

Alors, d'après le théorème $3, F / f_{0}$ est exceptionnelle au sens de Julia. Maintenant,

$$
\frac{F}{f_{0}}=1+\sum_{i=1}^{n} \alpha_{i} \frac{f_{i}}{f_{0}}=1+\sum_{i=1}^{n} \alpha_{i} g_{i}
$$

et la fonction

$$
\frac{F}{f_{0}}-1=\sum_{i=1}^{n} \alpha_{i} g_{i}
$$

est exceptionnelle au sens de Julia.

Puis, en vertu du théorème 1 , l'hypothèse entraîne que pour tout $i \neq j$

$$
f_{i} / f_{j}=g_{i} / g_{j}
$$

est exceptionnelle au sens de Julia.

5. Dans le cercle-unité. Dans ce paragraphe, on considère des analogues aux résultats donnés dans les paragraphes 2,3 et 4 pour le système défini dans le cercle-unité. 
Soit $f=\left(f_{0}, \cdots, f_{n}\right)$ un système dans $|z|<1$, c'est-à-dire, les fonctions $f_{0}, \cdots, f_{n}$ sont holomorphes sans zéros communs à toutes dans $|z|<1$.

DÉfinition 3. On dit que $J: \arg z=\theta_{f}$ est un rayon de Julia de $f$ s'il n'y a qu'au plus $2 n$ combinaisons des fonctions $f_{0}, \cdots, f_{n}$ linéaires, homogènes à coefficients constants, linéairement indépendantes $n+1$ à $n+1$ et exceptionnelles au sens de Picard dans $\Delta_{\varepsilon}\left(\theta_{f}\right) \cap(|z|<1)$, $\varepsilon$ étant positif quelconque.

THÉORÈme 7. Soit $f=\left(f_{0}, \cdots, f_{n}\right)$ un système dans le cercle-unité tel qu'au moins un rapport entre les fonctions $f_{0}, \cdots, f_{n}$ n'est pas normal. Alors, le système $f$ admet au moins un rayon de Julia.

Démonstration. D'après l'hypothèse, on peut supposer que $f_{1} / f_{0}$ $(\not \equiv 0)$ n'est past normale. Alors, il y a une suite $\left\{z_{k}\right\}_{k=1}^{\infty}$ contenue dans $|z|<1$ telle que

$$
\lim _{k \rightarrow \infty}\left(1-\left|z_{k}\right|\right) \rho\left(g\left(z_{k}\right)\right)=\infty
$$

où $\rho(g(z))$ est la dérivée sphérique de $g(z)$ ([4]). Soient

$$
z_{k}=r_{k} \exp \left(i \theta_{k}\right) \quad(k=1,2, \cdots)
$$

et $\theta_{0}$ un des points d'accumulation de l'ensemble $\left\{\theta_{k}\right\}_{k=1}^{\infty}$. Alors, on peut démontrer que $\arg z=\theta_{0}$ est un rayon de Julia de $f$ comme dans le cas du plan.

THÉORÈme 8. Soient $f=\left(f_{0}, \cdots, f_{n}\right)$ un système dans le cercle-unité sans rayon de Julia et

$$
F=\sum_{i=0}^{n} a_{i} f_{i} \quad(\not \equiv 0), \quad G=\sum_{i=0}^{n} b_{i} f_{i} \quad(\not \equiv 0)
$$

deux combinaisons linéaires à coefficients constants. Alors, la fonction $F / G$ est normale dans $|z|<1$.

En effet, on peut démontrer ce théorème comme dans la démonstration du théorème 3 .

Soient $g_{1}, g_{2}$ deux fonctions normales dans le cercle-unité. On sait que la somme

$$
g_{1}+g_{2}
$$


n'est pas normale nécessairement. Correspondant an cas du plan $|z|<\infty$, on donne ici une condition suffisante pour qu'une somme de fonctions normales le soit aussi.

Soient $g_{1}, \cdots, g_{n}(n \geqq 2) n$ fonctions normales, non-constantes dans le cercle-unité et $g$ un produit canonique des pôles des $g_{1}, \ldots, g_{n}$. (S'il n'y a pas de pôle, soit $g=1$.) On met

$$
f_{0}=g, f_{1}=g g_{1}, \cdots, f_{n}=g g_{n},
$$

alors, les fonctions $f_{0}, \cdots, f_{n}$ sont holomorphes sans zéros communs à toutes dans $|z|<1$. Dans cette situation, on a le

THÉORÈme 9. Si le système $f=\left(f_{0}, \cdots, f_{n}\right)$ n'admet pas de rayon de Julia, alors

$$
\sum_{i=1}^{n} \alpha_{i} g_{i}, \quad\left(\alpha_{i}\right) \neq(0)
$$

et

$$
g_{i} / g_{j} \quad(i \neq j)
$$

sont normales dans $|z|<1$.

On peut démontrer ce théorème comme dans le théorème 6 en utilisant le théorème 8 au lieu du théorème 3 .

\section{BIBLIOGRAPHIE}

[1] H. Cartan, Sur les zéros des combinaisons de $p$ fonctions holomorphes données, Mathematica, 7 (1933), 5-31.

[2] J. Dufresnoy, Théorie nouvelle des familles complexes normales; application à l'étude des fonctions algébroïdes, Ann. E.N.S., (3) 61 (1944), 1-44.

[ 4 ] O. Lehto et K. I. Virtanen, Boundary behavior and normal meromorphic functions, Acta Math., 97 (1957), 47-65.

[5] O. Lehto et K. I. Virtanen, On the behavior of meromorphic functions in the neighborhood of an isolated singularity, Ann. Acad. Sci. Fenn., 240 (1957), 1-9.

[6] R. Nevanlinna, Le théorème de Picard-Borel et la théorie des fonctions méromorphes, Gauthier-Villars, Paris, 1929.

[ 7 ] A. Ostrowski, Über Folgen analytischer Funktionen und einige Verschärfungen des Picardschen Satzes, Math. Zeit., 24 (1925), 215-258.

[ 8 ] H. L. Selberg, Algebroide Funktionen und Umkehrfunktionen Abelscher Integrale, Avh. Norshe Vid. Akad. Oslo, 8 (1934), 1-72.

[9] N. Toda, Sur une relation entre la croissance et le nombre de valeurs déficientes de fonctions algébroïdes ou de systèmes, Kodai Math. Sem. Rep., 22 (1970), $114-121$. 
[10] N. Toda, Sur les directions de Julia des fonctions algébroïdes dans $|z|<\infty$, Nagoya Math. J., 37 (1970), 53-60.

[11] G. Valiron, Sur la dérivée des fonctions algébroïdes, Bull. Soc. Math. France, 59 (1931), 17-39.

[12] T. Zinno, Some properties of Julia's exceptional functions and an example of Julia's exceptional functions with Julia's direction, Ann. Acad. Sci. Fenn., 464 (1970), 1-12.

Université de Nagoya 